\title{
PACIFIFA: A NEW MEASURE FOR EVALUATING AUTHORS' PRODUCTIVITY AND ACTIVITY OVER TIME
}

\author{
Asem Omari \\ Assistant Professor \\ College of Computer Science and Engineering Hail University, \\ Kingdom of Saudi Arabia \\ a.omarieuoh.edu.sa
}

\begin{abstract}
In this paper, we introduce a new measure for evaluating and characterizing the scientific output of a researcher over time. The PACIFIFA is a measure that takes different attributes into account in the process of evaluating the productivity, quality of research, and research activity of a researcher. This measure takes into account the number of the publications of the author, the sum of the citations of the publications, the H-Index of co-authors, the average impact factor of the host journals, the average impact factor of the research field of the author, and the time interval between the first and the last publication of the author. The advantage of this measure is that it gives an overview about the author's productivity, quality of publications, publications frequency over time, and the quality of the researcher(s) he/she worked with. In comparison to other measures, our measure showed a higher accuracy and reliability.
\end{abstract}

\section{KEYWORDS}

Author evaluation measure, New evaluation attributes, Author's productivity

\section{INTRODUCTION}

After the publication of a research work of an author or a researcher, It will be one of the key attributes to be considered in the evaluation of the success factors in the profile of the researcher. As we go through evaluating the profile of a researcher/author, we make a fast scan for the profile searching for the publications section. In that section, the number and types of publications the author have are investigated, the publication frequency over time and the co-authors of the researcher in order to find out if the author is a group worker or not. Furthermore, we may try to find out if this researcher has worked with other famous scientists or not, as this gives an indication of the quality of the research the researcher did.

In this paper we introduce a new measure that takes different attributes into account in the process of evaluating researcher's output quality. This paper is structured as follows: In the next section, we discuss some related work. In section 3, we introduce our proposed measure and discuss its calculation method. The summary and the future work are introduced in section 4 . 


\section{LITERATURE REVIEW}

The quality of research and researchers who conduct that research is an essential factor for almost all institutions, universities and companies, is considered as the key factor for hiring a new staff member, grant research fund, give the current staff new privileges or positions, put them in specific professional committees, and grant prizes and awards. Therefore, there should be a professional way that asset in the judgment process.

The quality of a research work and also the quality of the researcher who produces that research has been the research topic for a lot of researchers for the purpose of producing measures that evaluate the quality and the productivity of a researcher and the research works itself. Most of the evaluation process in those measures takes the number of publications produced by an author in addition to citation record for those publications.

The number of published papers gives an overview about the researcher's productivity, but it does not give an overview about the quality or the importance of those publications. The author in [1] proposed a measure, which he called the " $h$ index" to evaluate the quality of a researchers output. This measure represents the maximum number of papers that a researcher has published that have had at least the same number of citations [1]. For example, a researcher who has an hindex $=10$ means that he published at least 10 papers, each of which was cited at least 10 times. In effect, published papers with citations less than 10 do not count. The h-index does not take into account the quality of the co-authors [2][3], which is considered an important factor in evaluating the quality of the researcher because it reflects what people or what research groups he/she is involved in.

The impact factor is a measure of journal quality[4][9], This measure is used as the key factor to give important decisions such as whether to subscribe from a specific journal or not, or whether grant a research fund to an author who published his work in a specific journal with a minimum value of impact factor or not. The impact factors of journals increases continuously as the time goes on. Furthermore, impact factors vary with the variation of the research fields. For example, a journal of impact factor 2 in some academic field is considered high, but it may be considered low in other field. Figure 1, shows the difference of $H$-index as the research field differs. As shown in the figure the $h$-index of a number of physics researchers is much greater that the $h$ index of the same number of mathematics researchers. 


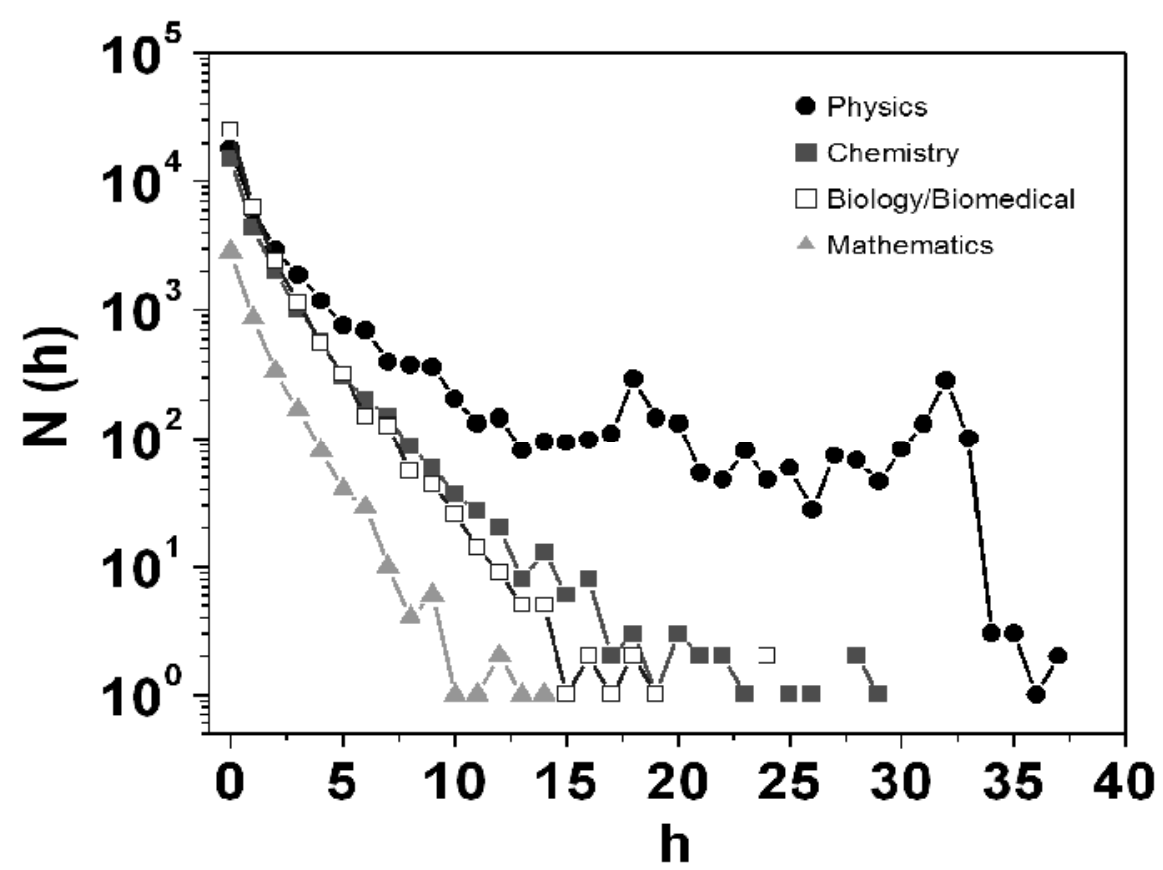

Figure1. Number of researchers with $h$ index in four different research fields (from [7])

According to [5] and [6], impact factor varies significantly according to subject field. Applied research areas have, in general, lower impact factor than fundamental or pure research areas. The variation is very significant in a way that the top journal in one field may have an impact factor lower than the bottom journal in another area.

The authors in [7], argued that, as the number of co-authors increase, the evaluation accuracy of the author based on the H-index measure will decrease, because the authors will cite their own publications and this will has its negative effect on the credibility of the results. Therefore, they introduced a new method that overcome this problem by proposing the $h I$ index to quantify an individual's scientific research output across disciplines.

The work in [10] discussed the need of the existence of different quality assurance measures and methods in order to evaluate people and researchers in an institution or university and assure a higher quality research output. The number of publications of a researcher gives an indication about the activity and productivity of the researcher. For example, a researcher who published 20 publications in a period of 7 years is absolutely more productive and more active than that of 5 publications in the same time period.

Distinguishing and highlighting the difference between a good and a bad research/researcher has also been a very hot research topic. The authors in [11], proposed ten factors that are essential, based on the authors point of view, for the researcher to succeed. Those factors include interest, motivation, inquisitiveness, commitment, sacrifice, excelling, knowledge, recognition, scholarly approach, and integration. In addition to research reliability and validity, the work in [12], argued that measures used to distinguish between a good or bad research should take into account the type of the research conducted. The authors classified any research type to be one of three main classes: Hypothesis-Testing and exploratory research, quantitative and qualitative research and basic and applied research. The paper in [13] discussed the importance of good research, common causes of bias, provided guidelines for evaluating research and data quality, and described examples of bad research. 
Therefore, we introduce a new measure for evaluating the quality and the productivity of a researcher that takes into account the researcher's publication record, the co-authors research quality, the impact factor of both the journal(s) published the research and the impact factor of the academic field, in addition to the time period in which the author published his/her research.

\section{Proposed Measure}

Our proposed measure $(P A C I F I F A)$ is calculated as follows:

$$
\text { PACIFIFA }=\text { Pub }+A_{J I F}+\frac{\left(\left(\text { Sum }\left(\text { CO }_{\text {HIndex }}\right)+\text { Sum }(\text { Citations })\right)\right.}{A_{F I F} * R_{A G E}}
$$

Where:

PACIFIFA: is the evaluation measure.

$P u b$ : is the number of publications of the author.

$A_{J I F}$ : is the average of the Impact Factors of the journals that published the research work where the impact factor (IF) of an academic journal is a measure reflecting the average number of citations to recent articles published in the journal.

$\mathrm{CO}_{\text {HIndex }}$ : is the sum of the $\mathrm{H}$-index of the co-authors. In order to generate an efficient evaluation of a researcher, the quality and productivity of the co-authors of the researcher should be taken into account because the quality of the researchers that a researcher works with reflects the research groups he/she works with. And thus reflects the quality level of the research done. The HIndex is both the productivity and impact of the published work of a scientist or a researcher. The index is based on the set of the researcher's most cited papers and the number of citations that they have received in other publications. A researcher with an index of $h$ has published $h$ papers each of which has been cited in other papers at leasth times [1]. As mentioned before, the HIndex reflects both the number of publications and the number of citations per publication. Sum(Citations): is the sum of the citations made to the publications of the author.

$A_{F I F}$ : Is the average of the impact factor of the field of specialization of the author e.g. computer science, Chemistry, Mechanical Engineering, Medicine, etc. This can be found in the ISI journal citation report [8], through indentifying the subject category.

$R_{A G E}$ : is the number of years from the date of first publication to the date of last publication. For example, if the first publication of the author was in 2001 and the last publication is in 2013, then the research age will be 2013-2001 $=12$. This factor will give an overview about the research activity of the researcher over time. Figure 2 below explains the attributes that are considered in the evaluation measure and how they are related to each other.

In order to evaluate this measure, we took the opinion of different faculty members in different fields at Hail University, Kingdom of Saudi Arabia. There was a positive opinion on the efficiency of this measure as it takes new and variable attributes into account in the evaluation process.

As a result, adopting this measure to evaluate authors' productivity and activity over time will have the advantage over other measures as it is more accurate and takes different attributes into account. Most of those attributes are not considered in other well-known measure. 


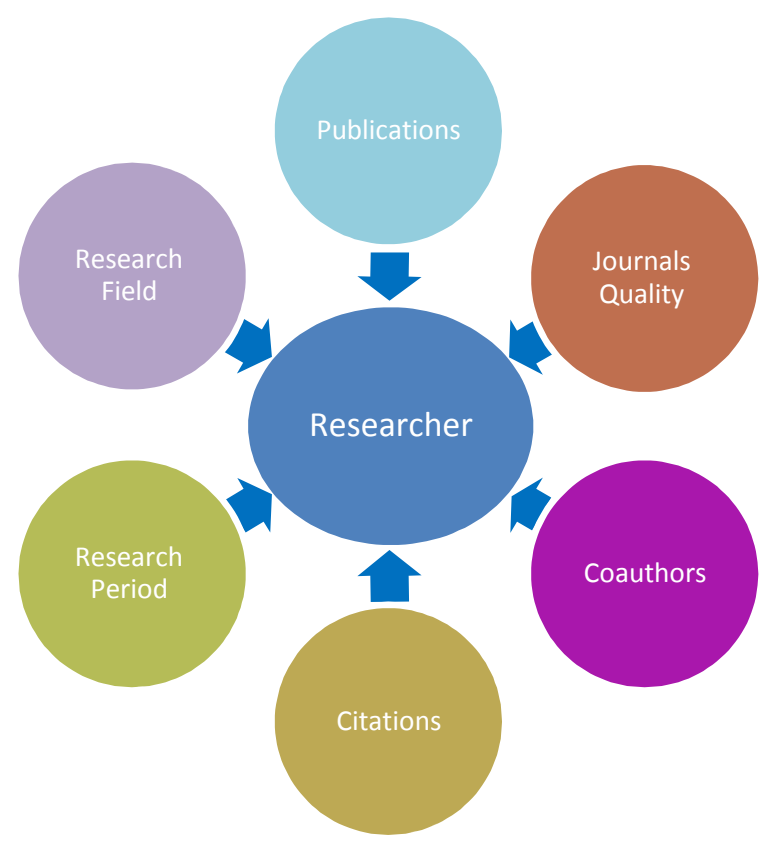

Figure2. Attributes affecting researcher's productivity and quality

\section{SUMmARY AND FUTURE WORK}

In this paper, we introduced a new measure to evaluate the productivity and quality of a researcher over time. This measure takes different attributes into account in the process of evaluating author's productivity and activity. Those measures include the number of the publications of the author, the sum of the citations of the publications, the H-Index of co-authors, the average impact factor of the host journals, the average impact factor of the research field of the author, and the time interval between the first and the last publication of the author. Those attributes represent the factors that have positive effect on a researcher's productivity and research quality.

As a future work we plan to make a comparison study between our proposed measure and other well-known measures. Furthermore, we plan to go further in improving the evaluation measure in a way that takes into account the type of the research produced by an author or researcher for example Hypothesis-Testing and exploratory research, quantitative and qualitative research and basic and applied research as mentioned in the literature review section above. Every research type will be given a relative weight to be considered and included in our evaluation measure. Thus, the evaluation accuracy will be improved. 


\section{REFERENCES}

[1] Hirsch, J. E. (15 November 2005). An index to quantify an individual's scientific research output. PNAS 102 (46): 16569-16572.

[2] Sekercioglu, Cagan H. (2008). Quantifying coauthor contributions. Science 322 (5900): 371.

[3] Zhang, C. -T. (2009). A proposal for calculating weighted citations based on author rank. EMBO Reports 10 (5): 416-417.doi:10.1038/embor.2009.74. PMC 2680883.PMID 19415071.

[4] Thomson Scientific (2013). THE THOMSON REUTERS IMPACT FACTOR, Retrieved april 30, 2013, from http://thomsonreuters.com/products_services/science/free/essays/impact_factor/

[5] Benjamin M. Althouse, Jevin D.West, and Carl T. Bergstrom, Theodore Bergstrom. Differences in Impact Factor Across Fields and Over Time, JOURNAL OF THE AMERICAN SOCIETY FOR INFORMATION SCIENCE AND TECHNOLOGY, 60(1):27-34, 2009

[6] Amin M., \& Mabe M. (2000). Impact factors: Use and abuse. Perspectives in Publishing, 1, 1-6. Andersen, H. (2000).

[7] PABLO D. BATISTA, MÔNICA G. CAMPITELI, OSAME KINOUCHI, ALEXANDRE S. MARTINEZ. Is it possible to compare researchers with different scientific interests? Scientometrics, Vol. 68, No. 1 (2006) 179-189.

[8] Thomson Reuters 2012. ISI Web of Knowledge. Journal citation reports (subject category summary). Last retrieved 25.05.2013 from: http://admin-apps.webofknowledge.com/JCR/JCR

[9] Garfield, E. (2005) 'The Agony and the Ecstasy - The History and Meaning of the Journal Impact Factor'. International Congress on Peer Review and Biomedical Publication, 2005, USA.

[10] Research Information Network. Quality assurance and assessment of scholarly research. A guide for researchers, academic administrators and librarians last retrived 20.05.2013 from www.rin.ac.uk/quality-assurance.

[11] Toledo-Pereyra LH 2012. Ten qualities of a good researcher. Journal of Investigative Surgery. Aug 2012;25(4):201-2. doi: 10.3109/08941939.2012.701543.

[12] Bill Altermatt 2010. Evaluating Research: How Can You Tell the Good Research from the Bad? Hanover college. From http://vault.hanover.edu/ altermattw/methods/assets/Readings/Evaluating_Research.pdf Last visited 15.05.2013.

[13] Todd Litman, Evaluating Research Quality Guidelines for Scholarship. International Electronic Symposium on Knowledge Communication and Peer Reviewing, International Institute of Informatics and Systemics (www.iiis.org), 2006.

\section{AUTHOR:}

Asem Omari: is an Assistant professor of computer Science at hail University, Kingdom of Saudi Arabia. Dr. Omari obtained his PhD. in Computer Science from Heinrich Heine University, Dusseldorf, Germany in 2008. He obtained a Graduate Certificate in Computer and Information Sciences from the University of Michigan/Dearborn, USA in 2002, and a bachelor degree in Applied Mathematics, System Analysis and Programming orientation from Jordan University of Science and Technology in 1999. His research interests include Database systems, Data Mining and knowledge discovery from

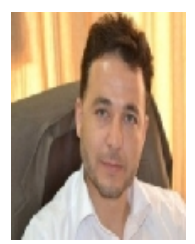
databases, e-commerce, e-learning and e-government. 